\title{
Irritable Bowel Syndrome
}

National Cancer Institute

\section{Source}

National Cancer Institute. Irritable Bowel Syndrome. NCI Thesaurus. Code C82343.

Gastrointestinal symptoms characterized by chronic abdominal pain and altered bowel habits in the absence of any organic cause. 